\title{
The use of Japanese Scalppuncture as a nursing intervention in patients with pain
}

\begin{abstract}
Background: Attending patients in pain is a common practice from the perspective of nurses; however many nurses have difficulty in acting autonomously in the control of the pain.
\end{abstract}

Primary study objective: To measure the perception of well-being with the use of Japanese Scalppuncture in patients in acute and chronic pain.

Methods/design: A multi focus case study was executed.

Setting: The study was conducted in Brazil and the method of Japanese Scalppuncture was applied. Participants: The sample consisted of participants, who presented a nursing diagnosis of acute pain or chronic pain.

Intervention: Different points were used, according to each patient's complaint, a total of 15 points were used.

Primary outcome measures: After the intervention, the level of self-perception of well-being increased from 3.1 to 9.7 in a 20 minute session. Due to our findings, we can infer that the procedure used collaborates with reduction of acute and chronic pain. After a Pearson correlation analysis, it was possible to identify that the accessory lateral point Dland $\mathrm{H}$ frontal point $\mathrm{H}$, both had a significant association $(\mathrm{p}<000,1)$ with the improvement of general well-being.

Results: $75.0 \%$ of the sample presented with "chronic pain" prevalent, and the defining characteristic (DC) that was more present was: "alteration in ability to continue previous activities" $(87.5 \%)$ and the related factor (RF) more prevalent in this diagnosis was: fatigue (75\%). The diagnosis "acute pain" was present in $25.0 \%$ of our sample, the DC most prevalent was "self-focused" $(87.5 \%)$, and the RF present in $100 \%$ of this subgroup was "physical injury agent".

Conclusion: it is perceived that the Japanese Scalppuncture is an effective care technology for intervention in patients with pain.
Volume 5 Issue 3 - 2018

\author{
De Oliveira Marcos Renato,' Martins Torres \\ Raimundo Augusto, ${ }^{2}$ De Freitas Maria Célia, ${ }^{2}$ \\ Da Silva Lúcia de Fátima, ${ }^{2}$ Guedes Maria \\ Vilani Cavalcante ${ }^{2}$ \\ 'Nursing professor, Federal University of Piauí, Brazil \\ ${ }^{2}$ Nursing professor, Ceará State University, Brazil
}

Correspondence: De Oliveira Marcos Renato, Nursing professor, Federal University of Piauí, Brazil, Tel +55 3422-3003, Email enfmarcosrenato@hotmail.com

Received: May 14, 2018| Published: June 19, 2018

Keywords: pain; acupuncture; nursing process

\section{Introduction}

The experience of pain by an individual, whether acute or chronic, is an event that causes a variety of disorders that in most cases have repercussions on negative events beyond the physical field. In the midst of clinical practice, it is perceived that pain aggravates other conditions. In general, pain causes feelings of vulnerability and helplessness, limits a person's daily activity, socially as well leisurely, tending to influence people's quality of life. ${ }^{1}$ Contemporary medicine has worked to find pathways and treatments to relieve or reduce pain experienced by people. Tremendous advances have been made in pain management, especially in the area of pharmaceuticals. ${ }^{2}$ However; the side effects associated with the use of pharmaceuticals products are a cause for concern. In the nursing context, the NANDA-I taxonomy ${ }^{3}$ established since 1986 the diagnosis of chronic pain (00133) and 10 years later NANDA-I published the diagnosis of Acute Pain (00132). But, it is noteworthy to note that the author Gordon ${ }^{4}$ has used the terminology "acute pain" as a nursing diagnosis since 1986. In addition to this historical context, it is underscored that pain is still a common diagnosis identified by nursing professionals. ${ }^{5}$
A review of literature demonstrates that the nursing diagnosis of acute pain is more common in patients in medical clinics ${ }^{6}$ and chronic pain occurs more frequently in patients in surgical clinics. ${ }^{7}$ This human response, pain, needs an adequate and effective therapeutic intervention, offering the minimum of risks and side effects. To assist, collaborating for a safe and resolute practice a nurse can use alternatives practices of care. An alternative practice aims to assist an individual in all aspects, whether in prevention, treatment or cure, considering the human as a holistic being. Among alternative caring practices, a nurse, in many countries, autonomously use elements of traditional Chinese medicine, such as acupuncture. There are many therapeutic indications for acupuncture, among them the treatment of pain. ${ }^{8}$ A differentiated technique, which has been used around the world, is the Japanese Scalppuncture. This technique main characteristic are the ease of application and low invasiveness, which reduces the risks. ${ }^{9}$ Thus, we perceive that acupuncture can be a caring technology that cooperates effectively in the nursing process. As a result, the present study aims to present the measures of the perception of well being, after the use of Japanese Scalppuncture in patients with acute pain and chronic pain. 


\section{Material and methods}

This is a clinical case study developed as part of the academic activities of the academic doctoral program in Clinical Care in Health, from the State University of Ceará (UECE), Northeast Brazil. A case study with multiple focuses and the classification of instrumental types were executed, that is, when the focus is not the case in itself, but a broader aspect. ${ }^{10}$ The broad aspect was an intervention using the Japanese Scalppuncture in patients with pain.

The sample consisted of eight participants, who presented a nursing diagnosis of acute pain or chronic pain, as described by Herdman \& Kamitsuru ${ }^{3}$ and were willing to participate in treatment through Japanese Scalppuncture as described by Enomóto ${ }^{9}$ and selected through a convenient process.

The collection of data was done through a clinical interview and physical examination, with analyzes of Capillary glucose, pulsation, blood pressure, waist and hip circumference; weight and height verification were performed to complement the information collected. The blood glucose measurement was done by means of a digital blood glucose meter and the blood collected by pricking the tip of the phalanges; the pulse count was made on a superficial artery, the abdominal circumference and hips were measured using a nonextendible measurement tape. The weight was measured on scale with $100 \mathrm{~g}$ graduation, with the person standing with both arms along the length of the body and the height measured with the scales ruler. The Body Mass Index (BMI) was calculated with the weight and height measurements. To minimize potential risks, the researchers received the participants in individual examinations rooms to ensure privacy.

It should be emphasized that the entire process of data collection, organization and analysis was validated by the Nursing Process as regulated by Brazilian law. The study also complied with the Resolution of the National Health Council, and was evaluated by the Ethics Committee of the State University of Ceará and received a positive number: 22739713.7.0000.5534. In order to present the results of the characterization data of the participants, descriptive statistics methods were used, such as relative risk, association and odds ratio, which made it possible for the visualization of the data obtained. The discussion of the data was based on the nursing theory of transpersonal care, a theory that proposes conscious intervention in care, enhancing healing and integrity. This theory does not rule out conventional science or modern nursing practices, but it complement them, which prioritizes above all the preservation of health and harmony of the human being. ${ }^{11}$ The data were transcribed using software SPSS version 21 and the measurements were described in terms of absolute frequency, followed by the association measures calculated according to the variable searched. The data were evaluated January of 2018.

\section{Results}

The perception of well-being was measured by using a Likert scale before and after the application of the procedure. On a scale of 1 to 10 , the closer to 1 the worse the individual perceived pain, while the closer to 10 on the scale the general feeling of well being improved. The mean the before the application was 3,1 and 9,7 after the application of the Japanese Scalppuncture. As a result, we can infer that the procedure used collaborated with the reduction of acute and chronic pain.
Regarding the presence of the nursing diagnoses (ND) relative to pain, $75.0 \%$ presented with "chronic pain" (00133), and the defining characteristics (DC) most present for this diagnosis were: "Alteration in the ability to continue previous activities" (87,5\%), and the "Facial expression of pain" (75\%), and the related factor (FR) most common in this diagnosis was: "Fatigue (75\%)". An addition diagnosis was "acute pain" (00132) present in $25.0 \%$ of the sample, the DC most presented was "self-focused" (87.5\%), and the RF "physical injury agent" was presented in $100 \%$ of this subgroup. After the physical examination and interview, each patient was placed in the supine position on a stretcher and the Japanese Scalppuncture was administered for 20 minutes. Different points were used according to each patient's complaints. A total of 15 points were used for a respective absolute frequency of: frontal (4), B frontal (4), AB frontal (1), C frontal, E frontal (1), H frontal (1), I frontal (1); B dorsal (1), C Dorsal (1), E Dorsal (1), D side accessory (3), D1 side accessory (1), F accessory (1), multiple points in VB (1); and NA point (1). After the Pearson correlation analysis, it was possible to identify that the D1 side accessory and $\mathrm{H}$ frontal were the points with significant association $(p<000,1)$ with the improvement of general well-being.

The public was predominantly female $(62.5 \%)$, with an average of 41.5 years of age, with a self reported skin color of black (75\%), working as general assistants $(75 \%)$. The data from the clinical interview and physical examination revealed that the majority had normal systemic blood pressure $(62.5 \%)$, and postprandial capillary glucose within the normal limits (87.5\%). Normal Body Mass Index (BMI) in $37.5 \%$ of the sample, but with some variations. It was possible to identify by the review of the waist hip ratio (WHR) that only $12.5 \%$ presented with a low risk of cardiac attack in the next 10 years, the majority of respondents were at moderate risk (25\%), high risk $(37.5 \%)$ or very high risk $(25 \%)$ of having a cardiac event with a strong negative impact in the next 10 years.

In our sample, only $25 \%$ used alcohol, but through statistical analysis it is possible to identify that consuming alcoholic beverages increases the risk of altering BMI by 4 times $(p<000,1)$. It was also identified that the sample was predominantly non-smokers $(87.5 \%)$ and the proportion of sedentary behavior was well balanced, that is, half of the sample was sedentary and the other half was non sedentary, but $50 \%$ is still a high level of sedentary life style. In the investigation of preexisting diseases, $75 \%$ stated that they had had some illness that required hospital admissions, and when questioned about their family history, the fact that the father had died of chronic disease $(50 \%)$, followed by father and mother (37,5\%).

\section{Discussion}

Pain is a warning symptom, common in multiple states with a negative impact on peoples' lives. While in pain, a client is often unable to recognize if there is change in an organ or if it is projected emotional alteration, so pain is a manifestation facet of the whole, which is the life of each person. Among several treatment options, acupuncture is sought after as a therapeutic resource used for pain management. ${ }^{12}$ The most common type of pain identified in this study was chronic pain, which is well supported by the literature, since a secondary clinical study ${ }^{13}$ confirms that chronic pain was the chief complaint seen in medical practice.

Acupuncture is embedded in Chinese holistic principles that govern as a preventive, curative technique and for the rehabilitation of several acute and chronic diseases. It was originally believed that 
acupuncture's effectiveness was due to its ability to manipulate $q i$, the energy of life. Studies examining the effects of acupuncture on chronic pain have demonstrated three primary locations of action, peripheral, spinal, and supraspinal. ${ }^{8}$ several studies have shown that most patients achieve significant pain reduction in their first session of acupuncture, and there is evidence that the use of acupuncture is effective in the continuous treatment of pain. ${ }^{8,14}$

In regard to the physiological action of acupuncture to pain, we emphasize that the academic literature provides sufficient data to affirm that acupuncture acts by physiological mechanisms independent of the placebo effect. Neurophysiology studies reveal that through the insertion of needles there is a stimulation of slower conducting and myelin-like C-type sensory fibers, also known as ameliorin, which carry the stimulus to the posterior horn of the spinal cord and ascends through spinothalamic tract. Acupuncture also promotes the release of a substance in the brain known as endorphins, which belongs to a subtype of neuropeptide called endogenous opioid and is an integral part of the natural mechanism of pain suppression. ${ }^{15,16}$

Also, it is well know that there are neuroprotective effects from acupuncture which includes vascular dementia; those effects are mediated through the reducing expression of Thioredoxin-interacting protein. ${ }^{17}$ The Japanese Scalppuncture technique is currently one of the most widespread acupuncture Microsystems; this system of acupuncture allows for the treatment of the whole body using only points located in the region of the head, practically painless and its effects are rapidly felt. ${ }^{9}$

However, it is difficult at the present time to compare the findings of this study with others. Specifically with a focus on the diagnoses researched, since most of the studies are primarily focused on the prevalence of these diagnoses, and not in nursing interventions. However, researchers have identified that nursing professionals often recommend the treatment of acupuncture for the improvement of the quality of life in people with musculoskeletal diseases. ${ }^{18,19}$

The finding from the patient's perspective will increase the knowledge about the effects of acupuncture, subsidize new proposals for research and interventions related to this subject, and serves as a subsidy for the indication of acupuncture as another alternative for those seeking relief from chronic or acute pain. We also believe that this client-oriented practice cooperates in the effective application of transpersonal caring theory. ${ }^{11}$

The analysis of the self-perception of well-being in this study was remarkable, because it was possible to identify almost immediately after the application of an acupuncture session that there was a significant improvement. Thus, if one considers the quality of life as a feeling of well-being in relation to oneself, in relation to fellowship with other people, and even with its own pathology, it is natural to suppose that everyone seek alternatives, besides traditional medicine, to achieve this well-being of healing symptoms such as pain. ${ }^{20}$ Another important point to highlight is that it was possible to identify that alcohol consumption is directly related to and a risk factor for a high BMI. This fact is in agreement with another recent study in which it also identified that alcohol consumption is associated with higher BMI values. ${ }^{21}$ Thus it is important that a nurse not only treat a symptom but also identify associated risks that may aggravate the present situation or even trigger other situations.

For example, it has been addressed that individuals who work in sedentary jobs, are three times more likely to have pain in more than one place. Pain that is predominant in regions such as the lumbar spine $(40.3 \%)$, the cervical spine $(27.3 \%)$ and shoulder $(23.8 \%){ }^{22}$ these regions are also cited as high indexes of pain points by individuals included in our study. Also it is important to identify not only if a client has pain but it is also necessary to use the appropriate point for the needle insertion, since the correct point locations are important contributors to the treatment effect. ${ }^{14}$ In our study two point, of all of the pointes showed a high level of self perception of wellness, the D1 side accessory and the $\mathrm{H}$ frontal point.

Finally, we point out that there are many gaps in the production of knowledge about the nursing process and its taxonomies especially in the effective of nursing care interventions especially with alternative technologies of caring. It has been described that acupuncture is effective treatment of chronic pain. The treatment effects of acupuncture persist over time and cannot be explained solely in terms of the placebo effect. So a referral for a course of acupuncture treatment is a reasonable option for a patient with chronic pain. ${ }^{14}$

In addition, additional studies are needed to understand the mechanism of action of acupuncture that presents satisfactory and practical results, whereas the contemporaneous model of science still has much to understand. Also, nurses need to review the applicability of tools for their patient population and other properties, such as reliability and validity. ${ }^{23}$ However, there are over 12,000 nonphysician acupuncturists in the United States and these practitioners are able to create a regimented treatment plans that has been shown to effectively manage pain in an otherwise complex and difficultto-treat conditions. ${ }^{8}$ We expect other studies will create additional treatment protocols, or even a physiological explanation of the events identified. ${ }^{24} \mathrm{~A}$ nurse must be highly competent, knowledgeable and possess positive attitudes towards pain management so that patients receive high quality pain management practices to facilitate optimal patient health outcomes..$^{25}$

\section{Conclusion}

The application of the nursing process requires nurses to master several skills and it is essential to follow a theoretical support system to better guide decision making which in turn should be shared wherever possible with the patient. This study allowed us to identify that pain, whether acute or chronic, generates suffering in the patient that goes beyond the physical aspects and that when a nurse has autonomous intervention practices, such as Japanese Scalppuncture, the results can be fast and satisfactory for the patient and it can also increase the selfperception of well-being by a client. We emphasize that the results found here have their limitations, common to the method used but it is necessary that this practice be shared for purpose of stimulating further research in this field.

\section{Acknowledgments}

None.

\section{Conflict of interest}

The author declares that there is no conflict of interest.

\section{References}

1. Kwissa-Gajewska Z, Gruszczyńska E. Relationship between daily pain and affect in women with rheumatoid arthritis: lower optimism as a vulnerability factor. Journal of behavioral medicine. 2018;41(1):12-21. 
2. Tracy S. Integrating Spiritual Assessment and Care in the Relief and Remission of Pain. Pain Management Nursing. 2017;18(1):1-2.

3. Herdman TH, Kamitsuru S. NANDA International nursing diagnoses: definitions and classification, 2017.

4. Gordon M. Manual of nursing diagnosis. Jones \& Bartlett Publishers. 2014.

5. Correia MDL, Duran ECM. Definição conceitual e operacional dos componentes do diagnóstico de enfermagem Dor Aguda (00132). Revista Latino-Americana de Enfermagem. 2017;25:e2973.

6. Mendes Bertoncello Fontes C, De Almeida Lopes Monteiro da Cruz D. Diagnósticos de enfermagem documentados para pacientes de clínica médica. Revista da Escola de Enfermagem da USP. 2007;41(3).

7. Soares Novaes E, Morbin Torres M, Vilcinski Oliva AP. Diagnósticos de enfermagem em clínica cirúrgica. Acta Paulista de Enfermagem. $2015 ; 28(1)$

8. Gurram S, Herati AS, Gilbert BR. The Role of Acupuncture in the Management of Chronic Pelvic Pain. Urological and Gynaecological Chronic Pelvic Pain.2017;85-93.

9. Enomóto J. Escalpopuntura japonesa: microssistema da nova acupuntura craniana. 1ed. São Paulo: Ícone; 2014.

10. Ventura MM. O estudo de caso como modalidade de pesquisa. Revista SoCERJ. 2007;20(5):383-386.

11. Willis DG, Leone-Sheehan DM. Watson's Philosophy and Theory of Transpersonal Caring. Nursing Theorists and Their Work-E-Book. 2017;66.

12. Lam WL, Yeung WF, Wong MK, et al. Combined electroacupuncture and auricular acupuncture for postoperative pain after abdominal surgery for gynecological diseases: study protocol for a randomized controlled trial. Trials. 2018;19(1):8.

13. Day MA. Defining Chronic Pain and its Territory. Mindfulness-Based Cognitive Therapy for Chronic Pain: A Clinical Manual and Guide. 2017;5-37.

14. Vickers AJ, Vertosick EA, Lewith G, et al. Acupuncture for chronic pain: update of an individual patient data meta-analysis. The Journal of Pain. 2017;19(5):455-474.
15. Menezes CRO, Moreira ACP, Brandão WDB. Base neurofisiológica para compreensão da dor crônica através da acupuntura. Rev dor. 2010;11(2):161-168.

16. Dimitrova A, Murchison C, Oken B. Effects of Acupuncture on Neuropathic Pain: A Systematic Review and Meta-analysis (P3. 306). Neurology. 2015;84(14):P3-306.

17. Du SQ, Wang XR, Zhu W, et al. Acupuncture inhibits TXNIP-associated oxidative stress and inflammation to attenuate cognitive impairment in vascular dementia rats. CNS neuroscience \& therapeutics. 2018;24(1):39-46.

18. Kurebayashi LFS, Freitas GFD, Oguisso T. Enfermidades tratadas e tratáveis pela acupuntura segundo percepção de enfermeiras. Revista da Escola de Enfermagem da USP. 2009;43(4):930-936.

19. Dias de Mello Pereira R, Titonelli Alvim NA. Delphi technique in dialogue with nurses on acupuncture as a proposed nursing intervention. Escola Anna Nery Revista de Enfermagem. 2015;19(1).

20. Ryan RM, Deci EL. Self-determination theory: Basic psychological needs in motivation, development, and wellness. Guilford Publications. 2017.

21. Abreu de Carvalho C, De Almeida Fonseca PC, Barbosa JB, et al. Associação entre fatores de risco cardiovascular e indicadores antropométricos de obesidade em universitários de São Luís, Maranhão, Brasil. Ciência \& Saúde Coletiva. 2015;20(2):479-490.

22. De Vitta A, Canonici AA, De Conti MHS. et al. Prevalência e fatores associados à dor musculoesquelética em profissionais de atividades sedentárias. Fisioterapia em Movimento. 2017;25(2).

23. McNett, Molly, Shelly Amato, et al. Sensitivity, specificity, and receiver operating characteristics: a primer for neuroscience nurses. J Neurosci Nurs. 2017;49(2):99-101.

24. Liu T, Yu JN, Cao BY, et al. Acupuncture for Primary Dysmenorrhea: A Meta-analysis of Randomized Controlled Trials. Altern Ther Health Med. 2017;23(7).

25. Karamjeet K. Knowledge and attitude regarding pain management among staff nurses. Nursing and Care Open Access Journal. 2017;2(1):1-3. 